\title{
Apropiación de las TIC en la educación superior: una mirada desde la disciplina del profesorado
}

\author{
Ana Teresa Morales Rodríguez ${ }^{1}$, Alberto Ramírez Martinell ${ }^{1}$, \\ Cora Beatriz Excelente Toledo ${ }^{2}$ \\ ${ }^{1}$ Universidad Veracruzana, Instituto de Investigaciones en Educación, Xalapa, Veracruz, \\ México \\ ${ }^{2}$ Laboratorio Nacional de Informática Avanzada, Xalapa, Veracruz, \\ México \\ ateremora@gmail.com, albramirez@uv.mx cora@lania.mx
}

\begin{abstract}
Resumen. En este artículo se hacen evidentes las diferencias observadas en los niveles de habilidad para el manejo de las Tecnologías de Información y Comunicación (TIC) y la frecuencia de uso que presentan los profesores universitarios de diversas disciplinas académicas. El interés particular de este estudio, radica en la relación que existe entre la naturaleza de las disciplinas (dureza o pureza) y el qué saben y qué tanto incorporan las TIC en sus actividades académicas los docentes de Educación Superior. Los resultados indican de manera general que los profesores cuentan con un nivel suficiente de saberes informáticos e informacionales, salvo algunas tonalidades marcadas por su disciplina. Asimismo se observa que existen diferencias en términos de frecuencia de uso de dispositivos y conectividad, de manejo de software para el apoyo de cada disciplina, y aprovechamiento de servicios que la institución educativa pone a su disposición.
\end{abstract}

Palabras clave: TIC, profesores en educación superior, saberes digitales, grado de apropiación tecnológica, disciplinas académicas.

\section{Introducción}

Es innegable que las Tecnologías de la Información y Comunicación (TIC) en la Educación Superior, inducen el cambio e impactan en el desarrollo económico y social de los países [1] y su incorporación es inminente lo cual se evidencia en la definición de políticas en las Instituciones de Educación Superior (IES).

En el caso de la Universidad Veracruzana (UV), esto se hace visible en los planes de desarrollo que los rectores han planteado desde finales de los años 90’s [2], a partir de los cuales se han dictado las estrategias y líneas de acción para la incorporación de las TIC, entre las que podemos mencionar: la dotación de infraestructura de TIC (equipamiento, conectividad, y servicios tecnológicos) para cada una de sus entidades académicas [3] (las cuales se encuentran distribuidas en cinco regiones geográficas); la implementación de programas de formación en TIC para el profesorado; la puesta en marcha de proyectos como AULA, en el que se capacitaron a miles de profesores para 
el uso de TIC tanto para el diseño de sus clases como para su ejecución; el establecimiento de un marco común de computación para todas las licenciaturas de la universidad; la ampliación de la oferta de servicios de TIC institucionales como iTunesUV, la biblioteca virtual, el sistema de información distribuida creado al interior de la universidad "Eminus", entre otros. Dado este contexto, es importante reflexionar que la incorporación de las TIC, implica no solo la dotación de infraestructura, sino que es necesario contar con habilidades para usarlas, y si bien es cierto que la universidad se ocupa de facilitar las TIC a la comunidad académica, es preciso conocer qué tanto son aprovechadas con fines académicos.

Ahora bien, es de notarse que la incorporación de las TIC a la Universidad no ha sido homogénea, tal como lo demuestran estudios realizados en diversas instituciones de educación superior a nivel internacional (Georgina y Olson, 2008; Johnson et al., 2012; Meyer \& Xu, 2009; Roberts et al., 2007 ) y nacional (Alarcón, López y Ortiz, 2013; Berra, 2012; Crovi, 2009; Sánchez y Prieto, 2012; Torres y Barona, 2012; Zubieta, Bautista, y Quijano, 2012), que desde diferentes enfoques y en distintas dimensiones han demostrado que existen brechas digitales en la incorporación de las TIC en la Educación Superior. Lo que hace asumir que al interior de la UV también existen brechas y en este estudio las observamos a partir del profesorado.

La brecha digital es un concepto no universalmente definido, que puede estudiarse desde múltiples dimensiones y observarse desde diferentes aristas. Es multifactorial y, para el caso de los profesores de las Instituciones de Educación Superior (IES), podemos señalar que la literatura ha estudiado este concepto a partir del efecto de factores como el grado académico, la edad, el género, la antigüedad, y la disciplina. Sin embargo, esta última no ha sido explorada a profundidad, aun cuando teóricamente se asume como elemento fragmentador de la profesión académica y del mismo sistema universitario [4]. Ya que, si la disciplina condiciona la conducta, prácticas, conjuntos de discursos, formas de pensar, procedimientos y motivaciones..."[5], tomamos como supuesto, que también condiciona -de alguna manera- las prácticas en el uso de las TIC; es decir, lo que necesitan utilizar (en términos de hardware y software); las formas de uso y su intención; su ideología o creencias acerca de la pertinencia -o no- del uso de las TIC en el contexto académico; y las motivaciones -o carencia de ellas- del uso de las TIC. Es por ello que esta investigación se ha planteado como objetivo, estudiar la relación existente entre la disciplina y el grado en que los profesores se apropian de las TIC dentro del contexto académico.

Para analizarlo y fundamentar lo anterior, se ha utilizado la taxonomía de Becher, que es una de las categorizaciones disciplinares más reconocidas y referenciadas en la literatura, la cual separa a las disciplinas según su dureza y pureza: Blanda-Pura, Blanda-Aplicada, Dura-Pura y Dura-Aplicada [6].

Cada una de de estas categorías presenta diferencias y características particulares, por ejemplo: las Blandas-Puras (p.e. Filosofía) se preocupan más por las cualidades, sus productos son el entendimiento y la interpretación, y están orientadas hacia las personas; las Blandas-Aplicadas (p.e. La enseñanza de Idiomas) son utilitarias, preocupadas por realizar práctica semi-profesional y sus resultados son protocolos y procedimientos; las Duras-Puras (p.e. Biología) se preocupan por asuntos universales, manejan hechos y datos cuantitativos, y sus resultados son descubrimientos $\mathrm{y}$ explicaciones; y por último, las Duras-Aplicadas (pe. Informática) tienen propósitos claros y específicos, son pragmáticas y sus resultados son productos y técnicas. Estas y 
otras características enmarcadas en la categorización, hacen asumir la hipótesis de que al existir diferencias en el uso de las TIC (brechas digitales), las disciplinas con carácter utilitario y pragmático (las Aplicadas) tienden a tener mayores niveles de habilidad en el uso de las TIC, y mayor frecuencia de uso, ya que sus fines son prácticos.

Así este artículo presenta como casos de estudio a profesores de cuatro licenciaturas, una por cada cuadrante de la taxonomía, todas con establecimientos ubicados en la región Xalapa de la UV. Específicamente, este análisis se realiza bajo un enfoque comparativo de dos elementos: el nivel de habilidad para el manejo de las TIC (saberes digitales informáticos e informacionales) y la frecuencia de uso de las TIC en el contexto académico, es decir que se provee información -hasta ahora no existenteacerca de qué tanto saben y qué tanto aprovechan las TIC de acuerdo a su disciplina, lo que puede apoyar el diseño de estrategias y el trazo de líneas de acción en las que la disciplina sea un factor determinante para la dotación de infraestructura y el fortalecimiento de habilidades de los profesores, para lograr una efectiva incorporación de las TIC en el contexto académico.

\subsection{Metodología}

Esta investigación forma parte del proyecto de Brecha Digital de profesores y estudiantes universitarios de la Universidad Veracruzana, y los ejes que le orientan están enmarcados en dicho proyecto1. La presente intervención se trata de un estudio de corte cuantitativo, basado en las comparaciones estadísticas de los saberes digitales [7, 8], frecuencia e intencionalidad de los profesores de cuatro licenciaturas; es observacional pues no se pretende manipular las variables sino observarlas en su contexto [9] y es transversal, pues se recabaron los datos en una sola temporalidad y no en diferentes momentos del tiempo. La técnica para la recolección de datos consistió en la aplicación de un cuestionario 2 a los profesores que impartieron clases en el periodo de febrero a agosto de 2014 y que completaron el instrumento en modalidad impresa y en el sistema en línea creado con este fin.

El estudio de caso, involucró a 108 profesores de los 186 que conforman el universo de las cuatro licenciaturas estudiadas (Se recabó información del $46 \%$ de los profesores de la Blanda-Pura, el 55\% de la Blanda-Aplicada, el 42\% de la Dura-Pura, y el 77\% de la Dura-Aplicada. Con respecto al género, el $57 \%$ son mujeres y el $43 \%$ hombres. Y por último, de acuerdo a tres rangos de edad generados en el software estadístico SPSS, el 32\% tiene entre 24 y 37 años, el 30\% entre 38 y 48 años, y el 38\% más de 48 años.

Para el análisis de la información, se construyeron índices en escala de 1 a 10, de cada saber digital [7], por ejemplo: de la habilidad para el manejo de dispositivos, archivos (locales y en línea), texto y texto enriquecido en procesador de palabras y administrador de diapositivas, datos en hojas de cálculo y software estadístico, y contenido multimedia; y otros relacionados con el manejo de la información, que en esta investigación se han denominado ciudadanía digital y literacidad digital. En el caso

\footnotetext{
${ }^{1}$ www.uv.mx/blogs/brechadigital/

${ }^{2}$ Diseñado al interior del proyecto de Brecha Digital en el periodo de Agosto 2012-Septiempre 2013, basado en una revisión de marcos de referencia de organismos como OCDE, UNESCO, ISTE, ILCE, entre otros. Con dos pilotajes previos a la versión final, y validado por la comunidad académica de la línea de investigación de Políticas en Educación Superior del Instituto de Investigaciones en Educación de la UV. Disponible en brechadigital.aexiuv.com/.
} 
de algunas otras variables como la cantidad de horas que utilizan los dispositivos móviles, se generaron categorías como de 0 horas (es decir que no lo usan), de 1 a 6 horas, y más de 6 horas, siempre diferenciando estos grados de uso por cada facultad. Para identificar las diferencias significativas entre los niveles de habilidad y frecuencia de uso de TIC de los profesores de las cuatro licenciaturas, se utilizó la prueba no paramétrica de Kruskal-Wallis.

\section{Resultados}

\subsection{Saberes digitales informáticos e informacionales de los profesores}

\section{Saberes digitales Informáticos}

En este tipo de saberes se considera el nivel de habilidad de los profesores para el manejo de dispositivos, archivos, texto y texto enriquecido, datos y contenido multimedia. Y respecto de cada saber, se encontró lo siguiente:

Dispositivos: Existen diferencias significativas $(\mathrm{kw}=.001)^{3}$, y se observa mejor nivel en la disciplina Dura-Aplicada (media= 8.5) arriba de la Dura-Pura (media=6.8); y de las Blandas, la Blanda-Pura (media=7.16) destaca sobre la Blanda-Aplicada (6.13). Ahora bien, las tareas que realizan con mayor habilidad en sus dispositivos, son: configurar un proyector (93\% pueden hacerlo); encontrar y ejecutar programas (87\%); Instalar programas (85\%); administrar antivirus (85\%); conectar y configurar impresora $(83 \%)$ y cámara $(82 \%)$; conectar un dispositivo de escritorio a Internet $(82 \%)$ y administrar actualizaciones $(81 \%)$. Además, en las que se encuentran profesores con menores habilidades para realizar cierto tipo de tareas, son las relacionadas con los dispositivos móviles como sin: conexión de dispositivos móviles a Internet (77\%), la sincronización de dispositivos móviles (69\%), y el mantenimiento general del sistema $(69 \%)$.

Archivos: En este rubro se encontraron diferencias significativas $(\mathrm{kw}=.014) \mathrm{Y}$ separando el manejo de archivos locales y archivos en línea, se encontró que el manejo de archivos en línea es lo que enmarca diferencias en los niveles de habilidad entre disciplinas. De los archivos locales, el 72\% tienen un nivel arriba de la media (8.5), lo que indica que los profesores cuentan con un nivel alto para realizar tareas como copiar, cortar, pegar, eliminar, renombrar, buscar, restaurar, ver propiedades, comprimir y descomprimir, y transferir, entre otras. En cuanto al manejo archivos en línea, la media es de apenas 5.5 y más de la mitad $(54.5 \%)$ de los profesores están por debajo de ésta. Además, las tecnologías más utilizadas para manejar archivos en línea, son: en primer lugar el correo electrónico (el 96\% de los profesores le manejan con niveles altos y medios); en páginas web, foros y blogs (59\%); en tecnologías de cómputo en la nube, como skydrive o google drive $(57 \%)$; y en redes sociales $(56 \%)$.

Texto y Texto Enriquecido: La media es de 7.17 y no hay diferencias significativas $(\mathrm{kw}=.109)$. En este rubro se revisa la habilidad para el manejo de texto en procesador de texto y administrador de diapositivas, y se observa que los profesores son

${ }^{3}$ Cuando el valor de la prueba de Kruskal-Wallis es menor a .05, la diferencia es significativa. 
ligeramente más hábiles para el manejo del procesador de texto que para el administrador de diapositivas.

Datos: La media es de 5.76 y este es el saber con mayores diferencias significativas $(\mathrm{kw}=.00)$, y quienes tienen mayor nivel de habilidad, son los profesores de la disciplina Dura-Pura (7.09); Dura-Aplicada (6.54); Blanda-Pura (5.31) y la más baja BlandaAplicada (3.9). Y al interior de esta variable, observamos que lo que incide en las diferencias del manejo de datos, es el manejo -o no- de software estadístico, pues las disciplinas de naturaleza Dura tienden a utilizar más datos de tipo cuantitativo, en relación a las disciplinas Blandas.

Contenido multimedia: En este saber, no se determinan diferencias significativas $(\mathrm{kw}=.127)$, sin embargo se nota que los profesores de la disciplina Dura-Aplicada tienden a ser más hábiles en el manejo de contenido multimedia. Así, en términos generales, las habilidades en que destacan los profesores, son el manejo de dispositivos multimedia, la transferencia de archivos multimedia y la frecuencia con que consultan contenidos en Internet y, con un nivel más bajo, en la conversión y edición de contenido multimedia. Por último, es evidente que a pesar del manejo que hacen de contenido multimedia, la integración -es decir la creación de contenido con previa planeación, y composición de audio, video e imágenes- de contenido, no es una habilidad que posean los profesores.

\section{Saberes digitales informacionales}

Para los saberes informacionales se examinan algunas prácticas de los profesores para el manejo de información y las categorizamos sea en el saber de literacidad digital cuando nos referimos a la aplicación de criterios de evaluación y selección de información y a la utilización de fuentes especializadas de acuerdo a su disciplina; y o en el saber de ciudadanía digital que considera las prácticas que reflejan responsabilidad para la garantía de integridad de la información, su seguridad y la cautela con que publican información personal en Internet, y por otro lado prácticas legales en el uso de la información (por ejemplo, piratería).

Literacidad digital: Las disciplinas Puras están por encima de las Aplicadas, y la más alta es Blanda-Pura, pues aplica mayores criterios para la selección de información. $\mathrm{Al}$ interior de este indicador podemos observar que los profesores poseen y practican buenos criterios para la selección de información (es decir para verificar que sea confiable, examinar distintas fuentes y analizar y evaluar la información), sin embargo -en todos los casos-, declaran no aplicar técnicas para realizar búsquedas especializadas en Internet (por ejemplo, uso de palabras claves, operadores booleanos, operadores de búsqueda, entre otros).

Ciudadanía digital: Son los profesores de la Blanda-Pura son quienes con menor frecuencia tienen buenas prácticas de nettiquete ${ }^{4}$, ejercen actividades que revelan responsabilidad en la integridad, seguridad, y cuidado de la información, así como algunos juicios de ética (principalmente en la descarga gratuita de películas). Ahora

\footnotetext{
${ }^{4}$ Uso adecuado del lenguaje formal e informal escrito en su forma gráfica, por ejemplo evitar el uso excesivo de emoticones o tipos de letra difíciles de leer.
} 
bien, con respecto a las demás disciplinas, la que tiene mejores prácticas de ciudadanía digital es la disciplina Dura-Pura, seguida de la Dura-Aplicada y la Blanda-Aplicada.

\subsection{Apropiación de las TIC en el contexto académico universitario}

Para efectos de este artículo, ya que lo que nos interesa es explorar evidencias del grado de apropiación de las TIC por parte del profesorado, nos enfocamos en la frecuencia con que los profesores utilizan las TIC para fines académicos (frecuencia e intencionalidad), y para esto se analiza la apropiación de: la computadora o laptop, dispositivos móviles, conectividad a Internet así como los servicios de TIC que pone a su disposición la universidad, y el software específico para cada disciplina.

Dispositivos: Considerando únicamente la frecuencia con que utilizan la computadora y laptop para fines académicos, encontramos que en general, los profesores tienden a usar más la laptop que la computadora y, las disciplinas que más la utilizan son las Duras (Aplicada, 90\% y Pura, 86\%) en comparación con las Blandas (Aplicada, $77 \%$ y Pura, $73 \%$ ).

Dispositivos móviles: aquí se examinó la frecuencia de uso del smartphone y la tableta para fines académicos. Respecto al smartphone se encontró que alrededor de la mitad de los profesores lo utilizan para fines académicos, las que menos le utilizan son las disciplinas Aplicadas (en la Dura-Aplicada lo usan un $42 \%$ y Blanda-Aplicada el 43\%); y de las Puras, en el caso de la Dura-Pura el 50\% lo usa con fines académicos, y en la Blanda-Pura el 55\%.

Internet: En este rubro, lo primero a resaltar, es que todos los profesores cuentan con acceso a Internet en las instalaciones de la UV y el $91 \%$ cuenta con servicio de Internet en casa, no así en el caso de la conectividad móvil, donde solo el $44 \%$ de los profesores cuenta con este servicio [10]. Lo que incide en el grado de apropiación (frecuencia de uso para fines académico), ya que se encontró que donde más se apropian de Internet es en su lugar de trabajo pues en el caso de todas las carreras, la frecuencia de conectividad dentro de la entidad académica es alta (medias entre 8 y 8.5 ); en segundo lugar está el hogar, donde se desataca que los profesores de la Dura-Aplicada. Por último, con respecto a la conectividad móvil, los grados de conectividad son bajos (medias entre 4.5 y 6.3).

Servicios de TIC institucionales: Los profesores de las disciplinas Puras, suelen usar un poco más los servicios institucionales (Dura-Pura $\mathrm{m}=5.71$ y Blanda-Pura $\mathrm{m}=5.43$ ), en relación a las disciplinas Aplicadas (Dura-Aplicada $\mathrm{m}=5.07$ y BlandaAplicada $\mathrm{m}=5.05$ ), aunque las diferencias no son significativas. Ahora bien, respecto a los servicios que más se utilizan, encontramos que el más utilizado es el servicio de correo electrónico en el que de acuerdo a la comparación de medianas, existe diferencia significativa $(\mathrm{m}=.037)$ destacándose los profesores de la disciplina Dura-Pura, quienes le usan con mayor frecuencia, seguidos de la Dura-Aplicada, Blanda-Pura y BlandaAplicada. El segundo servicio más utilizado, es el portal institucional, en el que no hay diferencias de uso marcadas por la disciplina. El tercer servicio más usado es la biblioteca virtual que refleja diferencias significativas $(\mathrm{kw}=.002)$ ya que en las disciplinas Puras (Blanda-Pura y Dura-Pura) hacen mayor uso, en comparación a las Aplicadas (Blanda-Aplicada y Dura-Aplicada) que la utilizan con menos frecuencia. 
Otro servicio que presenta diferencias de uso, es el sistema de información distribuida, en el que es evidente que los profesores que hacen mayor uso, son los de licenciaturas Duras, y dentro de las Puras, quienes menos lo utilizan son los profesores de la BlandaPura. En relación a los repositorios institucionales como iTunes, revistas de la UV, entre otros, los profesores que más los utilizan son los profesores de la Blanda-Pura y, respecto a los demás servicios (salas audiovisuales y blogs institucionales), la frecuencia de uso es baja y no se enmarcan diferencias de acuerdo a la disciplina.

TIC para efectos de comunicación y colaboración: Se encontró que en ninguna disciplina hay niveles altos de comunicación, la que más se comunica es la disciplina Dura-Aplicada, con una media de apenas 6.23, y en el caso de la colaboración, la disciplina que destaca es la Blanda-Aplicada con una media aún más baja (4.58). Ahora bien, al revisar con mayor profundidad estos indicadores, encontramos lo siguiente:

En cuanto a comunicación académica, las frecuencias son similares en todas las disciplinas, sin embargo las disciplinas Duras suelen publicar información en Internet con mayor frecuencia en comparación a las Blandas y donde las tecnologías que más se utilizan para fines de comunicación académica, es el correo electrónico, y no hay diferencia entre disciplinas; en segundo lugar, el whatsapp, y quienes más le usan con fines académicos, son los profesores de la Blanda-Pura y quienes menos, los profesores de la Dura-Pura; respecto a las redes sociales, observamos que los profesores de disciplinas Blandas las utilizan con mayor frecuencia que los de las disciplinas Duras; el chat en computadora, es mayormente usado en las disciplinas Aplicadas y menos usados en las Puras; las plataformas educativas como Eminus, son poco utilizadas, y quienes más la usa, son las Duras en relación a las Blandas; y por último, el medio menos utilizado, son los mensajes de texto — sms - en el contexto académico de los profesores.

Respecto a la colaboración académica, no hay diferencias significativas, sin embargo, las disciplinas que destacan son las Aplicadas, y en la que menos se colabora, es en la Blanda-Aplicada. Y por último, de acuerdo a lo declarado, los profesores que más utilizan las redes sociales para colaborar, son los de la Blanda-Aplicada, y los que menos le utilizan, son los de la Blanda-Pura.

TIC para prácticas propias de la disciplina: Es evidente que los profesores que tienden a utilizar más software especializado para su disciplina, son los de disciplinas Duras, ya que el $81 \%$ de de la Dura-Aplicada declaran utilizar software especializado y el $72 \%$ de la Dura-Pura, hacen uso de algún software para el apoyo de su disciplina $\mathrm{y}$, en el caso de las Blandas, es el carácter de aplicada la que diferencia el uso de TIC (43\% de los profesores de la Blanda Aplicada usan software en comparación con un $19 \%$ de la Blanda-Aplicada). Ahora bien, respecto al tipo de software que utilizan en cada disciplina, se realizará un análisis a mayor profundidad para futuras publicaciones, sin embargo, hasta el momento se observa al interior de cada disciplina que: los profesores de la disciplina Dura-Aplicada superan el número de software declarado en comparación a las otras disciplinas, e introdujeron software para la realización de tareas propias de la informática, tales como manejadores de base de datos, ambientes de desarrollo (programación), control de versiones, software estadístico, editores de imágenes y diagramas, entre otros; los profesores de la Dura-Pura señalan utilizar software para el análisis de datos (mediciones, graficaciones y estadística), geolocalización, procesamiento y edición de imágenes, entre otros; los de la Blanda- 
Aplicada señalan utilizar software para procesamiento de texto y texto enriquecido, reproducción de audio y video, así como para la edición de contenido multimedia; y por último, en la Blanda-Pura, la mayoría de los profesores no brindaron información acerca de software utilizado, y solo dos señalaron dos software relacionados con el procesamiento de texto (Latex y Acrobat).

\section{Conclusiones}

Respecto al papel de la disciplina en la habilidad para el uso de las TIC por parte de los profesores de las licenciaturas estudiadas en esta investigación, podemos concluir que la disciplina Blanda-Aplicada no destaca en los niveles de habilidad, caso contrario con la disciplina Dura-Aplicada, que en todas las habilidades analizadas tiende a destacar. Y en el caso del manejo de datos, la que destaca es la Dura-Pura debido a su naturaleza y tendencia al uso de datos cuantitativos. Sin embargo en términos de habilidades y prácticas en el manejo de información, el panorama es muy bueno, ya que las cuatro comunidades de profesores cuentan con niveles medios altos de habilidad. En términos de apropiación de las TIC, la aplicabilidad de las disciplinas, incide en un mayor uso de dispositivos, y frecuencia de uso de Internet para fines académicos. En lo que no destacan las aplicadas, es en la apropiación de servicios institucionales ya que les rebasan un poco las Puras, sin embargo es la Dura-Aplicada (Informática) donde se hace un mayor uso de sistemas de información distribuida. En el uso de las TIC para comunicarse y colaborar si favorece la aplicabilidad de la disciplina y por último, en el manejo de software especializado (de apoyo a su disciplina), no es la aplicabilidad lo que favorece la apropiación, sino la dureza, pues quienes hacen mayor uso de software especializado son la Dura-Pura y la Dura-Aplicada.

Dado este contexto y mecánica de trabajo, así como los resultados obtenidos, se cuenta con elementos para realizar análisis con mayor profundidad en cada uno de los elementos expuestos en este artículo, así como estar en condiciones de llevar a cabo estudios bajo perspectivas similares, pues hasta este punto de la investigación se ha hecho evidente la importancia que juega la disciplina en apropiación de las TIC de los profesores universitarios.

\section{Referencias}

1. Alarcón, E., Ortiz, V.: Estudiantes, profesores y TIC. La investigación en México, En Ramírez-Martinell, A., Casillas, A.M., Háblame de TIC: Tecnología Digital en la Educación Superior. 1a ed. Córdoba: Editorial Brujas (2013)

2. Crovi, D.: Acceso, uso y apropiación de las TIC en comunidades académicas. Diagnóstico en la UNAM, México, D.F.: Plaza y Valdés, (2009)

3. Kozma B.R.: National Policities that conect ICT-Based Education Reform to Economic and Social Developmet. Human Tecnology (2005)

4. PETIC-UV. Plan Estratégico de Tecnologías de Información y Comunicaciones, Dirección General de Tecnologías de Información. Recuperado el 15 de Abril, desde http://www.uv.mx/transparencia/files/2012/10/PlandeDesarrolloTecnologicoUV.pdf. (2012)

5. Universidad Veracruzana: Información Estadística, UV. Recuperado en Noviembre de 2014 de: http://www.uv.mx/informacion-estadistica/consultas- dinamicas/ (2014) 
6. Clark, B.: El sistema de Educación Superior una visión comparativa de la organización académica. Nueva imagen - Universidad Futura, UAM: México (1983)

7. Trowler, P., Knight P.: Departament-level Cultures and the Improvement of Learning and Teaching. Studies in Higher Education. Vol. 25, No. 1, Carfax Publishing (2000)

8. Becher, T.: Tribus y territorios académicos. La indignación intelectual y las culturas de las disciplinas Barcelona: Gedisa (2001)

9. Georgina, D.A., Olson, M.R.: Integration of technology in higher education: A review of faculty self-perceptions. Internet and Higher Education, Vol. 11, No. 1, pp. 1-8 (2008)

10. Johnson, T., Wisniewski, M., Kuhlemeyer, G., Isaacs, G., Krzykowski, J.: Technology adoption in higher education: Overcoming anxiety through faculty bootcamp. Journal of Asynchronous Learning Networks, Vol. 16, No. 2, pp. 63-72 (2012)

11. Meyer, K.A., Xu, Y.: A causal model of factors influencing faculty use of technology. Journal of Asynchronous Learning Networks, Vol. 13, No. 2, pp. 57-70 (2009)

12. Roberts, F., Kelley, C.L., Medlin, B.: Factors influencing accounting faculty members' decision to adopt technology in the classroom. College Student Journal, Vol. 41, No. 2, pp. 423-435 (2007)

13. Ojeda, M., Díaz, J., Apodaca, C., Trujillo, I.: Metodología de Diseño Estadístico. Xalapa, México: Universidad Veracruzana (2004)

14. Morales, A.T., Ramírez-Martinell, A.: Brecha digital de acceso entre profesores universitarios, de acuerdo a su disciplina. Revista Debate Universitario, Vol. 3, No. 6, pp. 149-158, República Argentina (2015)

15. Ramírez-Martinell, A., Morales, A.T., Olguín, P.A.: Marcos de referencia de Saberes Digitales. Edmetic: Revista de Educación Mediática y TIC, Vol. 4, No. 2 (2015)

16. Ramírez-Martinell, A., Casillas, M.A., Contreras, C.C.: La incorporación de las TIC a la enseñanza universitaria de los idiomas. Revista Debate Universitario, Vol. 3, No. 5, pp. 123 138, República Argentina (2014)

17. Torres, C., Moreno, G.: Inclusión de las TIC en los escenarios de aprendizaje universitario. Revista Innovación Educativa, Vol. 5, No. 1, Obtenido de http://www.udgvirtual.udg.mx/ apertura/index.php/apertura3/article/view/369 (2013)

18. Zubieta, Bautista, Quijano: Aceptación de las TIC en la docencia. Una tipología de los académicos en la UNAM, México: UNAM (2012) 